\title{
Virtual reality in neurorehabilitation-what matters? A therapists' view
}

\author{
Christopher Traenka ${ }^{1,2}$, Karin Wiesner ${ }^{1,3}$, Simone Albert ${ }^{1,3}$, Sandra Signer ${ }^{1,3}$, Philipp Mueller ${ }^{1,3}$, Theresa Fischer ${ }^{1,3}$, Matthias Frank ${ }^{1}$, Oliver \\ Mauthner ${ }^{3}$, Reto W. Kressig ${ }^{4}$, Nils Peters ${ }^{1,2}$ and Stefan T Engelter ${ }^{1,2 *}$ \\ ${ }^{1}$ Neurorehabilitation, University Department of Geriatric Medicine FELIX PLATTER, University of Basel, Burgfelderstrassse 101, 4002 Basel, Switzerland \\ ${ }^{2}$ Department of Neurology and Stroke Center, Department of Clinical Research, University Hospital Basel and University of Basel, Switzerland \\ ${ }^{3}$ Chief Nursing Officer, University Department of Geriatric Medicine FELIX PLATTER \& University of Basel, Basel, Switzerland \\ ${ }^{4}$ Chief Medical Officer and Chair of Geriatrics, University Department of Geriatric Medicine FELIX PLATTER \& University of Basel, Basel, Switzerland
}

\begin{abstract}
Objective: To evaluate which items matter for successful neurorehabilitation using virtual reality (VR)

Methods: We performed a single-center, explorative study in a neurorehabilitation unit. Five therapists, who used a commercially available VR-device (MindMotion Pro, MindMaze, Switzerland) for stroke patients with clinically relevant upper limb paresis in clinical practice, were asked to report their experience by filling in a standardised, pre-specified questionnaire. Variables included in this questionnaire were divided into 5 domains. Therapists ranked the variables' importance from 1 (unimportant) to 5 (very important). Results were given as mean scores \pm standard deviations.

Results: In total, 51 ( $\mathrm{n}=24$ female, age range 52-95 years) patients had VR-treatment (June 2017-January 2019). One fourth of the patients had $\geq 4$ sessions (range 1-14). All 5 therapists filled in the questionnaire with $100 \%$ completeness. The most important variables were, (i) usability of the VR-device with a mean score of 4.8 $( \pm 0.44)$ closely followed by (ii) patients' joy, an appropriate motor deficit and (iii) enough time for new tools, and easy handling, each with a mean score of 4.6 ( \pm 0.54$)$. Usage in individual one-to-one therapy sessions, as well as in circuit sessions was considered feasible, with the latter reaching a higher mean score (4.0 \pm 0$)$ than the former $(2.9 \pm 1.02)$. Unexpectedly, the patients` attitude towards technology $(2.6 \pm 0.54)$ and the therapists' interest in computers $(3.2 \pm 1.3)$ scored lower. Sex and age of the patients (i.e., $1.6 \pm 0.54 ; 1.2 \pm 0.54$ ) or the therapists ( $\operatorname{sex} 1.0 \pm 0$; age $1.8 \pm 1.3$ ) were considered unimportant for the success of VR-treatment.

Conclusion: Therapists considered self-explaining usability with easy handling and a short preparation time as most important determinants for successful usage of VR in neurorehabilitation. Patient characteristics focused on motivation and an appropriate motor deficit indicate the importance of an adequate patient selection. Age and sex did not matter. Even patients aged 90 years and older used the VR-device.
\end{abstract}

\section{Introduction}

There are an estimated 11'500 to 16 '000 patients with acute stroke in Switzerland per year [1,2]. In contrast to their acute treatment and the prevention of a secondary stroke, there is a substantial lack of high-level evidence about their rehabilitation. Stroke recovery is substantially determined by motor deficits, which are frequent and affect $>3 / 4$ of patients [3]. They matter to patients, since 6 months after stroke $2 / 3$ of patients are unable to incorporate the plegic hand in daily living [4], Solely approximately $1 / 3$ of stroke patients can walk one week after stroke. In turn, improvement of motor function is closely linked to better quality of life and is among the top priorities of affected stroke patients [5]. Given this high prevalence and tremendous burden, any measure to improve functional ability would be highly relevant for patients.

Virtual reality-based motor rehabilitation (VRbMR) is a relatively recent approach, which - according to a systematic review - shows evidence of a beneficial effect with regard to improving upper limb functioning and increased activities of daily living (ADL) if compared to conventional therapy without major safety concerns [6]. There are, however, currently still important barriers to its widespread use, including clinical usability [7]. Thus, knowledge is sparse regarding which factors matter for successful usage of VRbMR in clinical practice.
In particular, the views of the therapists and physicians involved have not been studied well although these are highly relevant for successful implementation. We addressed these questions by using a novel VRbMR system for task-specific upper extremity training after stroke, which has successfully been used in clinical practice [8]. The system offers interactive exercises integrating motor priming techniques and embodied visuomotor feedback.

\section{Methods}

\section{Setting}

We performed an explorative single-center, observational, study in a neurorehabilitation unit that is affiliated with an academic institution. Our neurorehabilitation unit is part of the established

${ }^{\star}$ Correspondence to: Stefan Engelter, Neurorehabilitation, University Department of Geriatric Medicine FELIX PLATTER, University of Basel, Burgfelderstrassse 101, 4002 Basel, Switzerland, E-mail: stefan.engelter@felixplatter.ch

Key words: Rehabilitation, recovery, virtual reality, stroke

Received: June 09, 2020; Accepted: June 16, 2020; Published: June 26, 2020 
stroke rehabilitation pathway of the stroke center at the University Hospital Basel. Key elements of this pathway are part of the national [9] and the European [10] certification process. Accordingly, after acute care, most stroke patients requiring in-hospital stroke rehabilitation are transferred to the neurorehabilitation unit of the university department of geriatric medicine FELIXPLATTER in Basel. The neurorehabilitation unit has 32 beds and is led by a neurologist as chief physician rehabilitation, a geriatrician, and staff neurologists, all experienced in stroke rehabilitation and with dual affiliations at both institutions. All patients receive active rehabilitative therapies, based on respective guidelines [11]. Patients with motor deficits are treated by therapists (physical (PT) and occupational therapy (OT)), who are trained in the principles of motor learning.

\section{Patients}

As part of their regular in-hospital rehabilitative treatment all stroke patients with a clinically meaningful deficit of the upper extremity could receive virtual reality rehabilitative treatment (VRbMR) for the upper extremities, if the following criteria were met: (i) Presence of at least some voluntary upper limb activities, (ii) capability to understand and follow the required tasks taking into account other neurological symptoms, cognitive impairment or the cardio-pulmonary condition, (iii) absence of epileptic seizures in the personal medical history. The decision whether an individual patient indeed received this treatment was left to the discretion of both the therapists (PT or OT) and the treating physician.

VRbMR treatment sessions lasted 30-45 minutes. Repetitive VRbMR treatments were possible as long as deemed appropriate to the patient by the therapists and the physicians and could be extended after discharge in an outpatient setting. This could include treatment sessions during day-care clinic ("Tagesklinik") or during outpatientrehabilitation sessions. If deemed appropriate also patients with neurological diseases other than stroke were allowed to participate.

\section{VRbMR}

The system used was MindMotion Pro (MindMaze SA, Switzerland), a VRbMR system developed to enhance rehabilitation of the upper extremity. The tasks and exercises are provided in gamelike scenarios [8]. The MindMotion PRO is a mobile platform that integrates an upper extremity motion capture technology (Figure 1). In this system, a screen placed in front of the patient displays an avatar that reproduces patient's movements in real time in a $3 \mathrm{D}$ virtual environment. A second touch screen enables the therapist to plan and customize exercises for upper limb motor rehabilitation. The exercises are based on activities of daily living, i.e. reach, grasp, or point, and engage patients' shoulder, elbow and wrist movements with various levels of difficulty [8].

The device includes a standard package of virtual reality exercises based on proven principles derived from neurorehabilitation and targeted at training motor skills at the different phases (acute, subacute and chronic) of the rehabilitation process after stroke or brain injury.

\section{Therapists}

In June 2017 two therapists, later in 2017 and early 2018 three additional therapists, i.e., in total 5 therapists (4 physical therapists and 1 occupational therapist) were instructed in the use of the device and used the device in their every-day clinical practice. In the last week of January and the first week of February 2019, all five therapists were asked to report their experience by filling in a standardised, prespecified questionnaire (Appendix).

\section{Questionnaire}

Therapists were asked to score the importance of pre-specified variables that may influence the likelihood of successful usage of virtual reality in stroke rehabilitation. Successful usage was arbitrarily defined as patient participation in all VR sessions offered, together with the impression of motor improvement either by the patient, or the therapist or both. Variables were divided in the following 5 domains: (A) patient's characteristics, (B) therapist's characteristics, (C) setting, (D) device for VRbMR, (E) neurologic disease.

Within each domain, the therapists were asked to rate the importance by numbers, which reflect the following meaning: $1=$ unimportant,

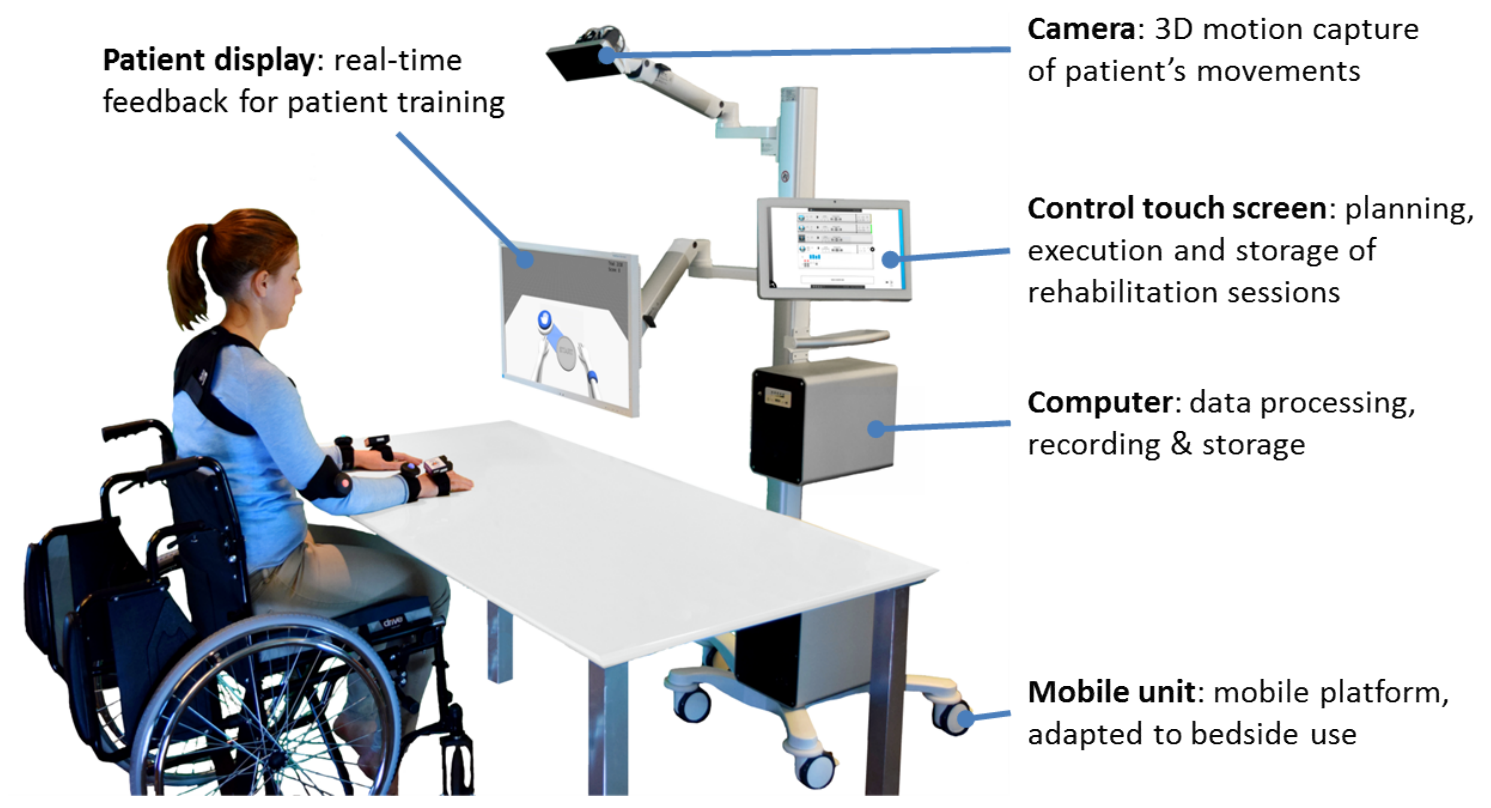

Figure 1. Overview of the MindMotionPRO and the main components (provided by MindMaze, who owns the copy right and agreed on the usage for this publication on June $5^{\text {th }}$, 2019). 
2=less important, $3=$ rather important, $4=$ important, $5=$ very important. Each number could be used as often as appropriate.

Furthermore, all therapists were encouraged to provide free text feedback on their general experience of the device regarding key benefits, constrains or limitation of use. The questionnaire was filled in and returned anonymously to the physicians (CT, NP, MF, STE), who performed the descriptive analyses, summarized the free-text feedbacks, and interpreted the findings.

\section{Statistical analysis}

Statistical analyses were restricted to descriptive analyses. For each pre-specified variable, means and standard deviations were determined.

Ethics: The design of the study did not require ethical approval as discussed with the Ehikkommission Nordwest- und Zentralschweiz EKNZ (June 18 $8^{\text {th }}, 2019$ ).

\section{Results}

From June 2017 to January 2019, in total 51 patients ( $\mathrm{n}=24$ female) received VRbMR-treatment. Patients' age ranged from 52 to 95 years with $>1 / 3$ being 80 years and older. Participants had $1-14$ sessions, with $1 / 4$ the patients receiving 4 or more sessions.

All 5 therapists (100\%) completed the questionnaire with $100 \%$ completeness.

The most important variables were, (1) usability of the VR-device which reached a mean score of 4.8/5 ( \pm 0.44$)$, closely followed by patients' fun or joy, an appropriate motoric deficit (i.e., patients deficits allow them to perform all the tasks and get challenged but not bored), enough time for new tools, and easy handling, each with a mean score of $4.6( \pm 0.54)$. Thereafter, therapists' joy and fun ranked next with a mean score of $4.0( \pm 0)$.

In contrast, sex, and age of the patients (i.e., $1.6 \pm 0.54 ; 1.2 \pm 0.54$ ) and those of the therapists (sex $1.0 \pm 0$; age 1.8 \pm 1.3 ) were considered less important.

Unexpectedly, the patients' attitude towards technology $(2.62 .6 \pm 0.54)$ and likewise the therapists' interest in computers $(3.2 \pm 1.3)$ scored low.
Likewise, the years of experience were rated unimportant, as long as the therapist were either a PT or OT and had an interest in novelties see (Figure 2). The therapists considered support by the superior less important than having enough time to explore and get used to new diagnostic tools (see figure 2). In the view of the therapists, VRbMR can successfully be used in individual one-to-one therapy sessions, as well as in group therapy settings, particularly within circuit session. However, the latter reached a higher score $(4.0 \pm 0)$ than the former (2.9 $\pm 1.02)$. Hemineglect $(3.6 \pm 1.15)$ was considered more important for the application than aphasia $(3.0 \pm 1.14)$. Although nearly all patients treated had a stroke $(4.4 \pm 0.88)$, therapists attributed Parkinson's disease $(4.3 \pm 0.57)$ and multiple sclerosis $(4.0 \pm 0.81)$ similar scores

With regard to the VRbMR device easy handling (4.6 \pm 0.54$)$, preparation time $(4.25 \pm 0.5)$ and variability regarding the tasks for the patients $(4.0 \pm 0.7)$ were all considered important (see figure 2). VRbMR-issues were most frequently addressed in the free text comments.

Free text comments focussed on the factor "time". In particular, the time required to (i) start the program or (ii) to install the sensors (iii) to navigate through the program were viewed as "time-consuming" and mentioned as reason for avoiding the usage of the VRbMR. Others mentioned that some aspects of reality, including haptic experiences were lacking in the virtual reality environment. Positive feedbacks mentioned that the VRbMR increased the level of activity of the patients, increased motivation of both patients and therapists.

\section{Discussion}

This explorative study addressed the importance of variables for successful use of VRbMR in neurorehabilitation from a therapists' perspective and revealed the following key findings: (i) easy, selfexplaining usability and handling as well as a short preparation time before using the device were considered most important, closely followed by variables characterizing the patients who are likely to benefit most: ideal motoric deficit as well as fun, joy and motivation for the therapy; (ii) regarding the setting, "enough time to explore and getting used to a new tool" was most important followed by fun and joy of the therapists; (iii) age and sex did not matter, neither for therapists nor for patients.

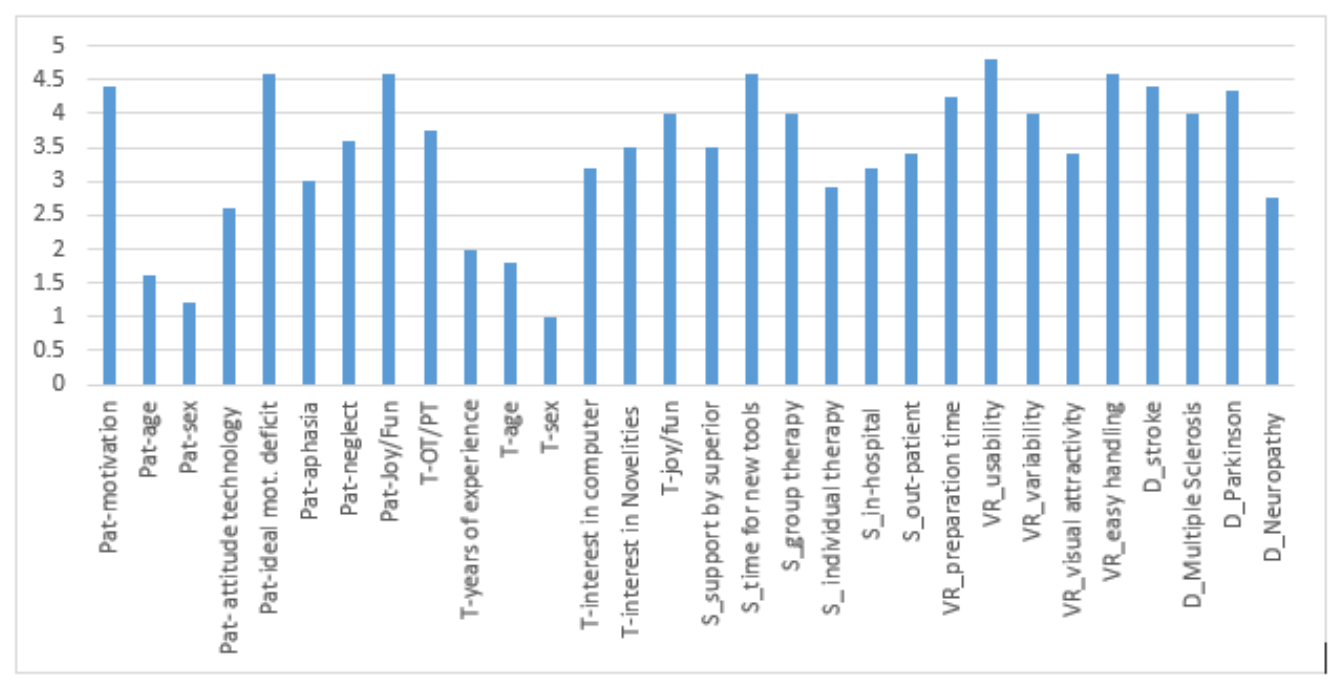

Figure 2. Therapists' ratings of the importance of predefined variables for successful usage of virtual reality in stroke rehabilitation.

Mean scores with the following meaning were displayed:1=unimportant, $2=$ less important, $3=$ rather important, $4=$ =important, $5=$ =very important. The following prefixes refer to the following domains. Pat_: patient characteristics, T_: therapist's characteristics, S_setting, VR_ Virtual reality device pro, D_ Diseases. 
All 5 therapists who were trained to use this VRbMR-device for 1.5 years indeed did use it in their patients. In addition, all 5 positively stated that they consider VRbMR a useful rehabilitative working tool in particular as an attractive "add-on"- to conventional therapy sessions. They also mentioned enjoying working with it and that also most of their patients had fun during the sessions too.

Nevertheless, according to a Cochrane Review, [6] VRbMR was used in solely $35 \%$ of the patients and in another study only $9 \%$ of the patients participating in stroke rehabilitation could be recruited for a VRbMR study [12]. This unexpectedly low rate of implementation is in line with the observation that just 51 patients in our neurorehabilitation unit had VRbMR in more than 1.5 years of usage.

Our observations indicate that in time of increasing pressure on institutions, physicians, and therapists to maximize rehabilitative therapy, the time for preparation of the VRbMR is not viewed as therapy time sensu strictu but as "lost time". Thus, any improvement for simplification and abbreviation of the preparation time is key for ensuring clinical utility, which has been mentioned a major barrier for its widespread use beyond research [7].

Although it has been thought that older patients might not be confident to use this technology [13], our observation showed that patients' age is not an issue. Interestingly, more than $1 / 3$ of our patients were aged 80 years and older and the oldest patient was even 95 years of age. Thus, the idea that virtual reality programs were more suitable for younger patients [7], seems outdated. Instead, age should not be viewed as a barrier for VRbMR as long as VRbMR fits the needs and key principles of modern rehabilitation in elderly patients [14].

Our observation stressed the importance of patient's motivation, joy, and fun. Although this seems expected, a recent review found no clear evidence between patient enjoyment and adherence as well as functional outcome [15]. At least, in a recent study in chronic stroke patients, which indicated a benefit in functional recovery by VRbMR, patients "liked to a great extent performing the exercises" [8].

As a novel aspect, we found that also therapists' fun and joy was important, although possibly less than that of the patients. However, the latter might rather reflect the therapists' general attitude of considering the patients' needs more important than their own. This adds to the previously shown importance of the interprofessional collaboration in VRbMR [16].

For hemispatial neglect, VRbMR was considered "rather important" by the therapists. This assessment is in line with current guidelines, which recommend that "it is reasonable to provide [among several other tools] ... virtual reality ... to improve neglect symptoms" as a Class IIa recommendation with an $\mathrm{A}$ as level of evidence [11]. Likewise, also in patients with aphasia of mild to moderate degree, i.e., communication about basic needs and wishes is present, therapists consider VRbMR rather important. Thus, in stroke patients with persevered communicative skills, the general recommendation that "virtual reality is reasonable to consider as a method for delivering upper extremity movement practice "(Class IIa recommendation with the level of evidence B) is not altered [11]. However, apart from other reason, the moderate level of evidence indicates the need for comprehensive, thoughtfully designed and adequately powered randomized controlled trials, before VRbMR can be considered standard of rehabilitative care. Studies like the current one might be helpful in designing such trials.

Strengths of our study include that - with the therapists' view we addressed an issue of both importance and one that has not been explored comprehensively yet. In addition, the fact that all therapists had completed the questionnaire without missing responses and that they provided additional free-text feedback increased the accuracy of the findings.

We are aware of several limitations: the number of therapists was relatively low. Thus, the generalizability of our findings remains unclear. Furthermore, we did not ask the patients about their views, which might have added another, yet very important perspective. Moreover, we did not assess functional outcome in relation to VRbMR. Thus, we are not able to study whether the variables considered important in the eyes of the therapists were associated with functional improvement.

In conclusion, from a therapists' perspective self-explaining usability, easy handling and a short preparation time are most important for the success of VRbMR in clinical practice. Patient characteristics focused on motivation and an appropriate motor deficit indicate the importance of a careful patient selection. Age and sex did not matter. Even patients aged 90 years and older used the VRbMR.

\section{Author contribution}

CT designed the study, analysed and interpreted the data, drafted the manuscript, and collected data. STE initiated, conceptualized, codesigned, and supervised the study, analysed and interpreted the data, drafted and revised the manuscript, and collected data. All authors: critical review of the manuscript, editing manuscript for content. All authors agreed on the submission of the present version of the manuscript.

\section{Conflicts of interest/disclosures}

Stefan Engelter (MD) has served on the scientific advisory board for MindMaze. MindMaze has provided the VRbMR device for the use in the institution free of charge. MindMaze had no influence of the study design, the analyses, the data collection, and the interpretation of the findings.

\section{References}

1. Gostynski M, Engelter S, Papa S, Ajdacic-Gross V, Gutzwiller F, et al. (2006) Incidence of first-ever ischemic stroke in the Canton Basle-City, Switzerland: a population-based study 2002/2003. J Neurol 253: 86-91. [Crossref]

2. Meyer K, Simmet A, Arnold M, H Mattle, K Nedeltchev (2009) Stroke events, and case fatalities in Switzerland based on hospital statistics and cause of death statistics. Swiss Med Wkly 139: 65-69. [Crossref]

3. Rathore SS, Hinn AR, Cooper LS, Tyroler HA, Rosamond WD (2002) Characterization of incident stroke signs and symptoms: findings from the atherosclerosis risk in communities' study. Stroke 33: 2718-2721. [Crossref]

4. Cramer SC (2015) Drugs to Enhance Motor Recovery After Stroke. Stroke 46: 29983005. [Crossref]

5. Miller EL, Murray L, Richards L, Zorowitz RD, Bakas T, et al. (2010) Comprehensive overview of nursing and interdisciplinary rehabilitation care of the stroke patient: a scientific statement from the American Heart Association. Stroke 41: 2402-2448. [Crossref]

6. Laver KE, George S, Thomas S, Deutsch JE, Crotty M, et al. (2011) Virtual reality for stroke rehabilitation. Cochrane Database Syst Rev 7: CD008349. [Crossref]

7. Laver KE, George S, Ratcliffe. J, Crotty M (2011) Virtual reality stroke rehabilitation-hype or hope. Aust Occup Ther J 58: 215-219. [Crossref]

8. Perez-Marcos D, Chevalley O, Scgmidlin T, GaripelliG, Serino A, et al. (2017) Increasing upper limb training intensity in chronic stroke uisng embodied virtual reality: a pilot study. J Neuroeng Rehabil 14: 119. [Crossref]

9. Arnold M, Engelter ST, Lyrer P, Renaud S, Michel P, et al. (2018) Certification of stroke centres and stroke units in Switzerland. Clin Translat Neurosci 1: 1-3.

10. Waje-Andreassen U, Nabavi DG, Engelter ST, Dippel DWJ, Jenkinson D, et al. (2018) European Stroke Organisation certification of stroke units and stroke centres. Eur Stroke J 3: 220-226. [Crossref] 
11. Winstein CJ, Stein J, Arena R, Bates B, R. Cherney L, et al. (2016) Guidelines for Adult Stroke Rehabilitation and Recovery: A Guideline for Healthcare Professionals from the American Heart Association/American Stroke Association. Stroke 47: e98-e169.

12. Edmans JA, Gladman JRF, Cobb S, Sunderland A, Pridmore T, et al. (2006) Validity of a virtual environment in stroke rehabilitation. Stroke 37: 2770-2775. [Crossref]

13. Lee J, Ku J, Cho W, Hahn W, Kim IY, et al. (2003) A Virtual reality system for the assessment and rehabilitation of activities of daily living. Cyberpsychol Behav 6: 383-388. [Crossref]
14. Engelter ST, Kressig RW (2018) Besonderheiten moderner Rehabilitation beim betagten Menschen. Praxis 107: 193-196. [Crossref]

15. Rose T, Nam CS, Chen KB (2018) Immersion of virtual reality for rehabilitation review. Appl Ergon 69: 153-161. [Crossref]

16. Schmid L, Glassel A, Schuster-Amft C (2016) Therapists' Perspective on Virtua Reality Training in Patients after Stroke: A Qualitative Study Reporting Focus Group Results from Three Hospitals. Stroke Res Treat 2016: 6210508.

Copyright: $\odot 2020$ Traenka C. This is an open-access article distributed under the terms of the Creative Commons Attribution License, which permits unrestricted use, distribution, and reproduction in any medium, provided the original author and source are credited. 